\title{
An Analysis of Infectious Disease Research Trends in Medical Journals From North Korea
}

\author{
Do-Hyeon Park' ${ }^{1}$ Min-Ho Choi ${ }^{2,3,4}$, Ah-Young Lim ${ }^{4}$, Hee Young Shin ${ }^{4,5}$ \\ ${ }^{1}$ Seoul National University College of Medicine, Seoul, Korea; ${ }^{2}$ Department of Parasitology and Tropical Medicine, Seoul National University College \\ of Medicine, Seoul, Korea; ${ }^{3}$ Institute of Endemic Diseases, Seoul National University Medical Research Center, Seoul, Korea; ${ }^{4}$ Institute for Health and \\ Unification Studies, Seoul National University College of Medicine, Seoul, Korea; ${ }^{5}$ Department of Pediatrics, Seoul National University College of \\ Medicine, Seoul, Korea
}

Objectives: This study aimed to investigate the current status of infectious disease research in North Korea by analyzing recent trends in medical journals from North Korea in comparison with research from South Korea.

Methods: Three medical journals (Preventive Medicine, Basic Medicine, and Chosun Medicine) were analyzed from 2012 to 2016. Articles on tuberculosis (TB), malaria, and parasitic diseases were selected and classified by their subtopics and study areas. Two medical journals published in the South Korea were selected for a comparative analysis of research trends.

Results: Of the 2792 articles that were reviewed, 93 were extracted from North Korea journals. TB research in North Korea was largely focused on multi-drug resistant TB and extrapulmonary TB, whereas research in South Korea more frequently investigated non-tuberculous mycobacteria. Research on parasitic diseases in North Korea was focused on protozoan and intestinal nematodes, while the corresponding South Korea research investigated various species of parasites. Additionally, the studies conducted in North Korea were more likely to investigate the application of traditional medicine to diagnosis and treatment than those conducted in South Korea.

Conclusions: This study presents an analysis of research trends in preventive medicine in North Korea focusing on infectious diseases, in which clear differences were observed between South and North Korea. Trends in research topics suggest a high prevalence of certain parasitic diseases in North Korea that are no longer widespread in South Korea. The large proportion of studies examining traditional medicine implies a lack of affordable medicine in North Korea.

Key words: Infectious diseases, Preventive medicine, Tuberculosis, Malaria, Parasitic diseases

\section{INTRODUCTION}

The long division between South and North Korea has resulted in major differences between the two societies, both in

Received: September 18, 2017 Accepted: March 13, 2018

Corresponding author: Hee Young Shin, MD, PhD Institute for Health and Unification Studies, Seoul National University College of Medicine, 71 lhwajang-gil, Jongno-gu, Seoul 03087, Korea E-mail: hyshin@snu.ac.kr

This is an Open Access article distributed under the terms of the Creative Commons Attribution Non-Commercial License (http://creativecommons.org/licenses/bync/4.0// which permits unrestricted non-commercial use, distribution, and reproduction in any medium, provided the original work is properly cited. terms of economics and healthcare. The life expectancy at birth of the South Korean population in 2015 was 82.3 years, while it was 70.6 years for North Korea, showing more than a 10-year gap, and the 2012 mortality rate of the North Korean population was 960.6 per 100000 population, which was 2.5 times higher than that of South Korea (389.1 per 100000 population) [1]. The greatest difference in the burden of disease between South and North Korea is exhibited in infectious diseases; the rate of disability-adjusted life years (DALYs) lost due to infectious diseases in North Korea in 2012 was 7319 personyears per 100000 population, which is more than 5 times higher than that of South Korea (1452 person-years) [1]. 
As suggested by many previous studies, tuberculosis (TB) is an infectious disease that requires extremely urgent attention and interventions in North Korea [2]. According to the World Health Organization (WHO), North Korea is one of the 30 nations with a high burden of TB and multidrug-resistant TB (MDR-TB) [3]. The TB incidence rate in 2016 in North Korea was 513 per 100000 population, which is much higher than that of South Korea (77 persons), the Southeast Asian population (240 persons), and the worldwide population (140 persons). However, caution should be taken when analyzing TB indicators, since other references suggest that the incidence of TB in North Korea has steadily increased: 345 persons per 100000 population in 2010, 429 in 2013, and 561 in 2015 [4]. Although efforts are being made by the international community to reduce the number of TB patients in North Korea, the issue of TB is not being adequately resolved due to repeated natural disasters and malnutrition, the difficulty of MDR-TB treatment, and the rise of extensively drug resistant-TB (XDR-TB) in North Korea [5].

Malaria and parasitic diseases are a major class of infectious diseases that the North Korean authorities and the WHO are paying attention to [2], and they are very relevant for the unification of Korea. Malaria in North Korea disappeared in the 1970s, but showed a rapid increase in the late 1990s, reaching total of 143674 cases in 2001. With international support, the number of patients decreased again, with 15392 patients recorded in 2010 and 5113 in 2016 [1]. Nonetheless, 9500000 people, or $37 \%$ of the North Korean population, live in active foci, and therefore require continued management [6]. Some have suggested that the malaria-transmitting mosquitoes in North Korea may travel to South Korea, directly impacting the occurrence of malaria in South Korea [7]. In fact, the indigenous malaria that had disappeared in the 1980s started to reemerge rapidly among soldiers stationed near the Military Demarcation Line, leading to 4193 patients becoming infected with malaria in 2000. The number of patients decreased to 673 in 2016, but the Gyeonggi, Incheon, and Gangwon areas that are adjacent to North Korea are still classified and managed as malaria risk areas $[1,7]$.

Parasitic diseases have not been investigated on a national level in North Korea, and it is difficult to assess the exact conditions because of the possibility of information control that prevents statistics from being disclosed. A previous study by Hong et al. [8] estimated the conditions in North Korea by investigating a sample of 236 residents living in North Ham- gyeong Province, near the border between the countries. The results of this study suggested that ascariasis and Trichuris trichiura infection rates were 43.2 and $40.3 \%$, respectively, which is equivalent to the rates in South Korea in 1976. Fortysix North Korean defectors living in China were examined for intestinal parasitic infections and similar results were reported, with rates of $41.4 \%$ for ascariasis and $37.6 \%$ for T. trichiura [9]. In a 2006 investigation by the Korea Centers for Disease Control and Prevention (KCDC) examining 1501 North Korean defectors, intestinal parasites were detected in 434 people (28.9\%) [10]. There is a risk of parasites spreading upon the unification of Korea when human and material resources are actively exchanged, and South Korean physicians' lack of experience may postpone the diagnosis or result in misdiagnosis. Therefore, it is important to accurately understand and continue to manage parasitic diseases in North Korea before inter-Korean exchanges increase $[8,11]$.

The previous studies discussed above focused on understanding the situation based on statistics provided by external organizations; however, they were limited due to the lack of primary sources of data and samples. As a result of the difficulties in securing credible data, many South Korean researchers have estimated the circumstances in North Korea on the basis of empirical data collected through testimonies or survey results from North Korean defectors; however, this methodology is also limited because it is difficult to arrive at generalizations based on those data, and this area of research has focused on mental and social health issues, rather than physical diseases [12]. To supplement the methodological limitations of previous healthcare research on North Korea, some studies have investigated the interests and needs expressed by the North Korean authorities based on the North Korean literature.

Yi et al. [13] and Lee et al. [14] analyzed the conditions of medical technology and preventive medicine in North Korea using medical textbooks, and found a lack of content in epidemiological research methodology, especially regarding infectious diseases. Kim [15] analyzed the In-Min-Bo-Gun (People's Health), which was used as a medium to promote the Hygiene Reform Movement; this was the first attempt at analyzing a North Korean medical journal. Huh [16] analyzed 497 papers published in Chosun Medicine and reported that the most frequently mentioned disease groups were digestive, cardiovascular, and neoplasmtatic diseases and that many of the studies were on diagnosis and treatment. Kim and Noh [17] analyzed the studies published in Preventive Medicine between 
1982 and 2006 and found that most of the studies were related to infectious diseases, and according to Jung et al. [18], who analyzed the keywords of the same journal, most studies were focused on experimental research into bacterial detection, immune system maintenance, and infection prevention. In an attempt to take a closer look at trends in clinical medicine, Kim [19] focused on digestive, cardiovascular, respiratory, and endocrine diseases by analyzing a specialty-specific journal, Internal Medicine, while Choi et al. [20] investigated trends in research on TB and respiratory diseases. Even so, research on North Korean medical journals is still lacking, especially since no study has yet systematically examined trends in comprehensive medical research journals specific to certain diseases, and no study has compared these trends to those in South Korea.

The analysis of medical journals yields insights into the current status of research by providing information on the academic interests within a society, and can also provide an indirect understanding of the level of medical development by investigating the use of technological equipment in the studies [17]. As a socialist state, North Korea's main propaganda agen$\mathrm{da}$ and major political tasks at a given time are reflected in issues from the corresponding year of publication [21]. Furthermore, since North Korea emphasizes the importance of preventive medicine in its Socialist Constitution and Public Health Act [17], it can be concluded that North Korean healthcare authorities' interests and priorities on preventive medicine are reflected in comprehensive medical research journals. This study, therefore, aimed to analyze how North Korea understands and manages its internal healthcare issues from the perspective of preventive medicine, with a particular focus on diseases that are prioritized, such as TB, malaria, and parasitic diseases, through the research trends found in the comprehensive North Korean medical research journals. Furthermore, a comparative analysis was conducted between these research trends and those of South Korea to characterize the different conditions of infectious diseases in South and North Korea and to propose tasks that the field of healthcare needs to address in preparation for unification.

\section{METHODS}

This study conducted a descriptive analysis of research trends on infectious diseases (TB, malaria, and parasitic diseases) in South and North Korean medical journal publications in the last 5 years. Data collection and analysis were conducted for 4 months, during the periods of January-February and June-July, 2017.

\section{Data Collection}

The research subjects were selected from North Korean medical journals obtained through the Ministry of Unification's Information Center on North Korea. Of the 9 available journals, the 3 that were comprehensive medical research journals were chosen: Preventive Medicine, Chosun Medicine, and Basic Medicine. According to the Chosun Encyclopedia, each of these journals is a comprehensive medical journal in the fields of preventive medicine, medicine, and basic medicine, respectively, with their main readership being researchers, professionals, healthcare workers, and educators [22]. By selecting comprehensive medical research journals, not journals specific to specialties within clinical medicine, this study aimed to analyze the overall emphasis placed on infectious diseases, especially the overall research trends for TB, malaria, and parasitic diseases. The scope of the project was 2792 studies published in a total of 180 issues during the Jong Un Kim period of 2012-2016. Taking into account the possibility of a researcher from North Korea submitting their papers overseas during the same period, PubMed was searched for authors with an affiliation including "Democratic People's Republic of Korea." This search yielded 43 studies, and as was the case in preceding studies [23], only 1 of those articles investigated infectious diseases [24], meaning that PubMed was of limited use for understanding infectious disease research trends in North Korea.

In order to compare the research trends between South and North Korea, the Journal of Preventive Medicine and Public Health (JPMPH) was initially selected to be analyzed. However, since the research topics covered in the JPMPH were more diverse than those of North Korean journals, with proportionately fewer studies focusing on the 3 diseases of interest, it was difficult to claim that they represented trends in infectious disease research in the South Korea. Instead, Tuberculosis \& Respiratory Diseases (TRD) and the Korean Journal of Parasitology (KJP) were chosen because they allowed a comparison of these specific 3 classes of diseases. These journals are the only Korean medical journals that specialize in the field of TB and parasitology and are listed in the Korean Citation Index. The time period of the analysis was congruent to that of the North Korean medical journals (2012-2016), and 439 studies pub- 
lished in a total of 44 issues were selected from TRD and 520 studies published in a total of 28 issues were selected from KJP.

\section{Data Analysis}

All data on the papers' authors, titles, year of publication, and references were recorded using Microsoft Excel (Microsoft Corp., Redmond, WA, USA). Two researchers individually searched the titles of all 2792 North Korean publications and extracted 337 studies on all infectious diseases, and from those studies, further extracted 93 studies related to TB, malaria, and parasitic diseases. Of the 959 South Korean publications that were initially identified, 71 from TRD were selected, excluding studies conducted by foreign authors and research conducted on respiratory diseases, and 223 from KJP, excluding research conducted by Korean researchers but on foreign countries such as those in Southeast Asia.

Two frameworks for analysis were applied to the studies selected from the title search. The first framework was a classification by diseases, with reference to the Korean guidelines for TB and the International Statistical Classification of Diseases and Related Health Problems, 10th revision. In this framework, studies were classified as dealing with pulmonary TB, extrapulmonary TB, MDR-TB, latent TB, non-tuberculous mycobacteria (NTM), and others. The categorization was conducted by dividing the studies into those related to Mycobacterium TB (MTB) and those related to NTM. Then, the studies on MTB were divided into resistant bacteria and susceptible bacteria and the studies on susceptible bacteria were further divided into pulmonary TB, extrapulmonary TB, and latent TB based on the form of infection. Malaria and parasitic diseases were subdivided according to their biological classification: helminths, protozoa (including malaria), and arthropods. Helminths were further categorized into nematodes, trematodes, and cestodes. The second framework was a classification by field of study: epidemiology, diagnosis, treatment, home remedies, prevention, case reports, experimental research, and others $[16,17]$. Two researchers conducted all research extraction and classification, and when differing opinions were presented, the final decision was made by consensus. The results from each categorization were collected, Excel was used to calculate the frequency, percentage, and average for each category, the results were compared to the trends in articles from South Korean journals, and these findings were described, focusing on the differences. Lastly, the results of the analysis were com- pared to those of previous research (Figure 1).

\section{RESULTS}

\section{Tuberculosis}

Research on pulmonary TB was the most common topic in both South and North Korea, with 29 publications from South Korea (41\%) and 17 from North Korea (50\%), but some important differences were found. North Korean journals published 8 studies (23\%) on MDR-TB and 6 on extrapulmonary TB $(18 \%)$, which were higher proportions than observed in the South Korean journals. In contrast, there were 3 publications on NTM (9\%), which is a much smaller proportion than was observed in the journals from South Korea (34\%) (Figure 2). The results for the classification by field of study showed that most research was done on diagnosis and treatment, each of which accounted for 10 of the publications for North Korea (29\%), and 5 studies (15\%) investigated home remedies, which is a higher rate than was observed in the journals from South Korea. Case reports (3\%) and epidemiological studies (6\%) were, however, less common than in the journals from South Korea (Figure 3).

There were only 2 studies of the epidemiology of TB in North Korea, making it impossible to collect data on the prevalence of pulmonary TB; however, our results confirmed the frequency of NTM. In a study published in Preventive Medicine (2015), 340 sputum specimens were collected from patients with respiratory diseases before they had anything to eat. NTM species were identified at the following rates: Mycobacterium fortuitum, 46.1\%; M. kansasii, 26.9\%; M. abscessus, 15.4\%; and M. avium complex (MAC), $11.5 \%$. In a South Korean study, the bacteria isolated from the positive sputum specimens in pulmonary disease patients were as follows: MAC, 32\%; M. abscessus, 29\%; M. fortuitum, 17\%; and M. kansasii, 2\% [25]. M. kansasii, which was rare in South Korea, was common in North Korea, while the most common NTM in South Korea, MAC, was infrequent in North Korea.

The number of studies on the diagnosis of TB was relatively high. Most of the studies on drug-resistant TB involved the use of molecular diagnosis, while the research on pulmonary TB simply introduced existing diagnostic methods in the format of a review article. According to the article entitled "Up-to-date diagnosis of pulmonary TB and evaluation of disease activity" published in Preventive Medicine (2012), the current tests that are run are clinical manifestations, the erythrocyte sedimenta- 


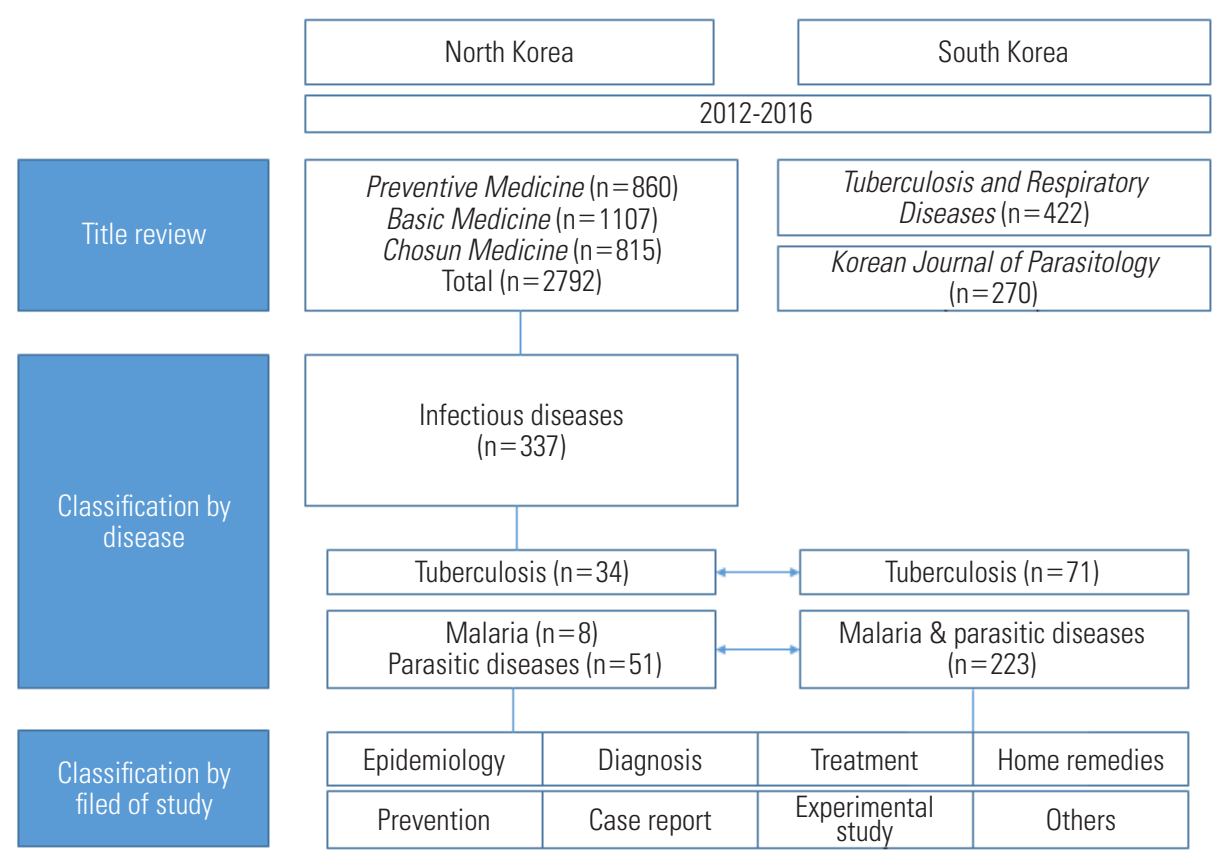

Figure 1. Flowchart of analysis process.

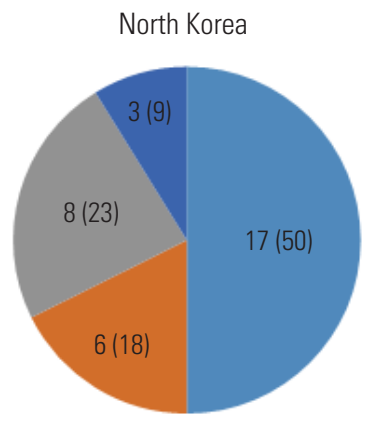

- Pulmonary tuberculosis

- Extrapulmonary tuberculosis

- Multidrug-resistant tuberculosis

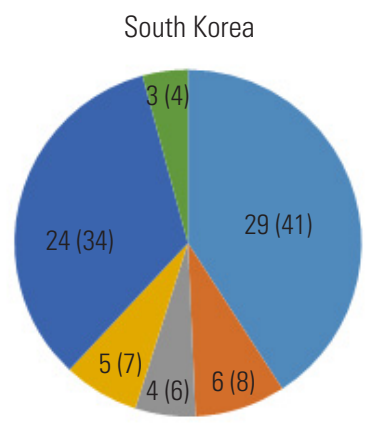

- Latent tuberculosis

- Non-tuberculous mycobacterial infection - Others

Figure 2. Comparison of studies on tuberculosis between North and South Korea (classification by disease). Values are presented as number (\%).

tion rate, and acid-fast stain (Ziehl-Neelsen stain); the study states that in order to overcome the limitations of the current tests, biochemical, immunological, and serum tests are being researched [26]. Such methods are already used universally in South Korea, and smear tests and culture have replaced TB DNA amplification methods.

Research into the treatment of TB showed a trend for experimental treatments to be investigated. Drugs that seem to have been self-developed in North Korea, such as an anti-TB oil emulsifier drug and a combined injection of hydroxypropyl

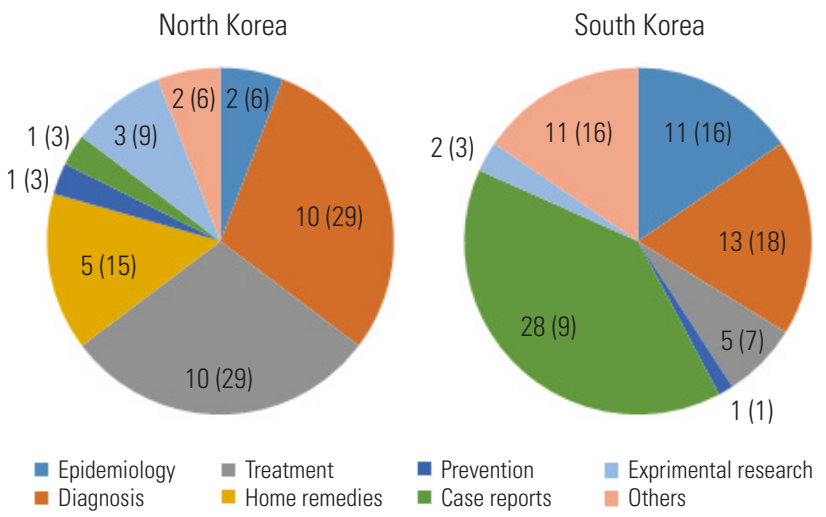

Figure 3. Comparison of studies on tuberculosis between North and South Korea (classification by field of study). Values are presented as number (\%).

$\beta$-cyclodextrin and anti-TB agents, and experimental methods, such as ozone-water mixtures, were attempted for treatment. Research into these modalities was conducted with the goal of using these methods on patients who are difficult to treat with available drugs, such as patients in whom treatment failed due to the presence of MDR-TB or side effects. Furthermore, several studies aimed to increase the effectiveness of TB treatments or to reduce the duration of medication and side effects by combining natural products with previously existing TB treatments, such as combining rifampicin 
and chitosan, ethionamide/ciprofloxacin and extracts from Phellodendron amurense fruit, and TB treatments and Japanese knotweed.

\section{Malaria and Parasitic Diseases}

Of North Korean studies investigating parasites, including malaria, $34(57.6 \%)$ were on protozoa, 8 on nematodes (13.6\%), 7 on trematodes (11.9\%), and 4 on cestodes (6.8\%). Eight papers investigated malaria. In comparison, 71 of the studies collected from the South Korean journals were on protozoa (31.8\%), and 13 were on malaria. The percentages of studies on nematodes, trematodes, and cestodes were similar between North and South Korea. Although only 14 types of parasites were studied in North Korea and no research was conducted on arthropods, there was some research on parasites that were not studied in South Korea (Table 1). North Korea showed high proportions of studies focused on diagnosis (39\%), experimental studies (22\%), and treatment and home remedies (16\%). As with TB, there was less research on epidemiology and case reports than in the articles from South Korea, but a greater focus on diagnosis and treatment (Figure 4).

\section{Malaria}

Although only 8 studies on malaria were published in North Korea, they included review articles and short communication introducing international research or established theories that allowed an indirect look into the status of malaria in North Korea. According to Basic Medicine published in 2013, "The current problems related to malaria are the lack of effective pre- ventive medicine, the development of drug-resistant protozoa, and outbreaks of insecticide-resistant vectors" [27]. As this study suggests, North Korea is also seeing an increase in antimalarial drug-resistant Plasmodium species. All research conducted in North Korea focused on $P$. vivax malaria, while 1 review introduced $P$. knowlesi malaria. No research was found on P. malariae malaria or P. falciparum malaria.

North Korea seems to be automating and advancing diagnostics by implementing polymerase chain reaction (PCR) and the use of stereomicroscopy camera-computer systems. However, the abovementioned study in Basic Medicine stated that "The biochemical analyses commonly used in hospitals are not useful in identifying the biochemical characteristics of Plasmodium," indicating weaknesses in extant diagnostic methods [27].
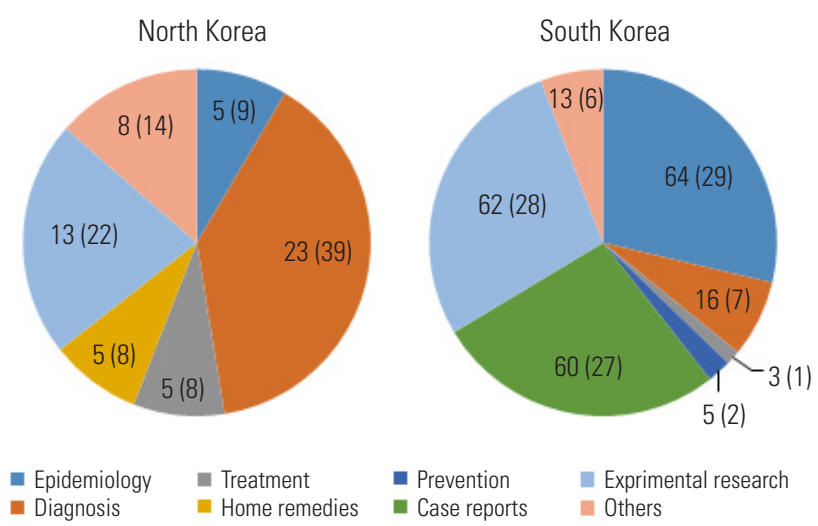

- Exprimental research - Diagnosis = Home remedies - Case reports

Figure 4. Comparison of studies on malaria and parasitic diseases between North and South Korea (classification by filed of study). Values are presented as number (\%).

Table 1. Comparison of studies on malaria and parasitic diseases between North and South Korea (classification by species)

\begin{tabular}{|c|c|c|c|c|}
\hline \multirow{2}{*}{ Classification } & \multicolumn{2}{|r|}{ North Korea } & \multicolumn{2}{|r|}{ South Korea } \\
\hline & $\mathbf{n}(\%)$ & Species & n (\%) & Species \\
\hline \multirow[t]{10}{*}{ Nematodes } & $2(25.0)$ & T. canis & $8(17.0)$ & T. canis, Toxascaris leonine \\
\hline & & & $7(14.9)$ & Trichinosis, trichinellosis, Trichinella spiralis \\
\hline & $3(37.5)$ & Ascariasis & $6(12.8)$ & Ascaris suum \\
\hline & & & $6(12.8)$ & Anisakidosis, anisakiasis, A. simplex, A. pegreffii \\
\hline & & & $3(6.4)$ & $\begin{array}{l}\text { Strongyloidiasis, St. stercoralis, } \\
\text { St. myopotami }\end{array}$ \\
\hline & & & $3(6.4)$ & Enterobiasis, Enterobius vermicularis \\
\hline & & & $2(4.3)$ & Capillariasis, Capillaria hepatica \\
\hline & & & $2(4.3)$ & Dirofilariasis, Dirofilaria immitis \\
\hline & & & $2(4.3)$ & Gnathostomiasis, Gnathostoma spinigerum \\
\hline & $2(25.0)$ & Hookworms & & \\
\hline
\end{tabular}




\section{Journal of}

Preventive Medicine \& Public Health

Table 1. Continued from the previous page

\begin{tabular}{|c|c|c|c|c|}
\hline \multirow{2}{*}{ Classification } & \multicolumn{2}{|r|}{ North Korea } & \multicolumn{2}{|r|}{ South Korea } \\
\hline & n $(\%)$ & Species & n $(\%)$ & Species \\
\hline & $1(12.5)$ & Trichius trichiura & & \\
\hline & & & $8(17.0)$ & $\begin{array}{l}\text { Others (Bourgelatia diducta, Cosmocephalus obvelatus, } \\
\text { Globocephalus samoensis, Schistorophus } \\
\text { cirripedesmi, Gordius sp., thelaziasis, nematodes [2]') }\end{array}$ \\
\hline Subtotal & $8(13.6)$ & & $47(21.1)$ & \\
\hline \multirow[t]{7}{*}{ Trematodes } & & & $6(17.1)$ & Fascioliasis, F. hepatica, F. gigantica \\
\hline & $7(100.0)$ & Clonorchis sinensis & $6(17.1)$ & Clonorchiasis, Clonorchis sinensis \\
\hline & & & $4(11.4)$ & $\begin{array}{l}\text { Echinostome, Echinostoma cinetorchis, } \\
\text { Chaunocephalus ferox }\end{array}$ \\
\hline & & & $4(11.4)$ & $\begin{array}{l}\text { Pygidiopsis summa, Haplorchis taichui, Acanthotrema } \\
\text { felis }\end{array}$ \\
\hline & & & $2(5.7)$ & Gymnophalloides seoi \\
\hline & & & $2(5.7)$ & N. sp., N. seoulense \\
\hline & & & $11(31.4)$ & $\begin{array}{l}\text { Others (paragonimiasis, Metagonimus yokogawai, } \\
\text { Microphallidae, Macroorchis spinulosus, Cercaria } \\
\text { caribbea, intestinal fluke [2]', Trematode [4]') }\end{array}$ \\
\hline Subtotal & $7(11.9)$ & & $35(15.7)$ & \\
\hline \multirow[t]{5}{*}{ Cestodes } & & & $10(34.5)$ & Sparganum, sparganosis, Sp. erinacei, Sp. decipiens \\
\hline & & & $7(24.1)$ & D. latum, D. nihonkaiense \\
\hline & $1(25.0)$ & Cysticercosis & $3(10.3)$ & Ta. saginata, Ta. asiatica, taeniasis \\
\hline & $3(75.0)$ & Echinococcus granulosus & $3(10.3)$ & Echinococcosis, hydatid, E. multilocularis \\
\hline & & & $6(20.7)$ & $\begin{array}{l}\text { Others (Hymenolepsis nana, Nybelinia surmenicola, } \\
\text { Mesocestoides lineatus, Catenotaenia dendritica, } \\
\text { Ligula intestinalis, Oswaldotrema nacinovici) }\end{array}$ \\
\hline Subtotal & $4(6.8)$ & & $29(13.0)$ & \\
\hline \multirow[t]{8}{*}{ Protzoa } & $9(26.5)$ & Toxoplasma & $20(28.2)$ & Toxoplasmosis, Toxoplasma gondii \\
\hline & $8(23.5)$ & Malaria & $13(18.3)$ & Malaria, P. vivax, P. falciparum, P. ovale \\
\hline & $1(2.9)$ & Trichomonas & $12(16.9)$ & Trichomoniasis, Trichomonas vaginalis \\
\hline & $8(23.5)$ & $\begin{array}{l}\text { En. histolytica, En. dispar, Acanthamoeba, Naegleria } \\
\text { fowleri, Balamuthia mandrillaris }\end{array}$ & $10(14.1)$ & $\begin{array}{l}\text { Acanthamoeba, Acanthamoeba castellanii, } \\
\text { En. histolytica }\end{array}$ \\
\hline & $4(11.8)$ & Cryptosporidium & $3(4.2)$ & Cr. spp., Cr. hominis \\
\hline & & & $2(2.8)$ & G. lamblia, G. intestinalis \\
\hline & $1(2.9)$ & Cyclospora & & \\
\hline & $3(8.8)$ & Others & $11(15.5)$ & $\begin{array}{l}\text { Others (Tritrichomonas foetus, Thelohanellus kitauei, } \\
\text { Isospora belli, Myxobolus sp., leishmaniasis, Azumio- } \\
\text { bodo hoyamushi, Eimeria tenella [2]', Encephalitozo- } \\
\text { on cuniculi, Pneumocystis jirovecii, Babesia gibsoni) }\end{array}$ \\
\hline Subtotal & $34(57.6)$ & & $71(31.8)$ & \\
\hline \multirow[t]{3}{*}{ Arthropods } & & & $15(60.0)$ & $\begin{array}{l}\text { Ticks, Caparinia tripilis, otodectosis, Panonychus citri, } \\
\text { Amblyomma testudinarium, Haemaphysalis } \\
\text { longicornis, Acarapis mite, Ornithodoros sawaii }\end{array}$ \\
\hline & & & $6(24.0)$ & Culicoides spp., flies, Lucilia sericata \\
\hline & & & $4(16.0)$ & $\begin{array}{l}\text { Others (Haemadipsa rjukjuana, Aedes togoi, } \\
\text { cephalonomia gallicola, chewing lice) }\end{array}$ \\
\hline Subtotal & $0(0)$ & & $25(11.2)$ & \\
\hline Others & $6(10.2)$ & & $16(7.2)$ & \\
\hline Total (n) & 59 & & 223 & \\
\hline
\end{tabular}

T, Toxocara; A, Anisakis; St, Strongyloides; F, Fasciola; N, Neodiplostomum; Sp. Spirometra; D, Diphyllobothrium; Ta, Taenia; E, Echinococcosis; En, Entamoeba; Cr, Cryptosporidium; G, Giardia; P, Plasmodium.

${ }^{1}$ The numbers in parentheses refer to the number of articles. 


\section{Parasitic diseases}

Only 5 studies investigated the epidemiology of parasitic diseases in North Korea. However, a glimpse into the conditions in North Korea was provided by a study on T. trichiura and hookworms that have not emerged in South Korea. A study entitled "Epidemiological study of biliary ascariasis" published in Preventive Medicine, researched 96 patients with biliary ascariasis [28]. Since ascariasis has almost disappeared in South Korea, this number is quite high.

Some epidemiological studies were conducted on Clonorchis sinensis, Toxoplasma gondii, and amoebas, with each topic having 7, 9, and 8 publications, respectively. The higher percentages of studies on these issues in North Korean research indicate that these are prevalent conditions in North Korea.

Although the conditions of hydatids in North Korea are unknown, the 3 studies on hydatids were all on Echinococcus granulosus. Hydatidosis in South Korea is mainly caused by $E$. granulosus, but all case reports have suggested transmission from foreign countries [29]. In contrast, case reports from North Korea state that it is the cases were indigenous. Furthermore, Cryptosporidium was commonly found in North Korea's water and environment [30] and Cyclospora was a common cause of diarrhea in patients at day care centers, preschools, and caregivers [31].

Diagnostic studies on parasites in North Korea mainly investigated diagnostic methods, such as serum tests and molecular genetic tests, that are still difficult to universally perform in North Korea. Most of the studies attempted molecular genetic diagnostics and identification using PCR for diagnostic purposes, but according to a study in Basic Medicine [32], "Although there are interests in new diagnostic technologies such as PCR, this method is only possible in research institutes that have abundant reagents and equipment; therefore, this method cannot be introduced more broadly," suggesting that the application of research into practice was difficult. On the other hand, there was no diagnostic research on helminths, especially on the diagnosis of intestinal parasites. This may be because diagnosis is easy through fecal exams.

Investigation into the research on the treatment of parasitic diseases showed that the 3 studies focusing on ascariasis were all on treatment and prevention. One study discussed the impact of the pro-hygiene propaganda and the other 2 were on traditional Korean medicine, such as warm oxygen injections and box moxibustion treatment with the SaGunJa combination. Although studies stated that praziquantel was used to treat $C$. sinensis, this is unlikely because the current conditions make it difficult to produce the drug domestically. Research into the treatment of protozoiasis showed many attempts to replace metronidazole, including treatment strategies using traditional Korean such as Cnidium monnieri and leek juice, well as research on Trichomonas and comparisons between metronidazole and the antiprotozoal effects of Agrimonia pilosa extract.

\section{DISCUSSION}

The results of this study provide indirect insights into the conditions of the main healthcare issues-TB, malaria, and parasitic diseases-in North Korea. The fact that TB, malaria, and parasitic diseases comprise a high percentage (27.6\%) of research into infectious diseases shows that North Korea's healthcare priorities are reflected in medical research trends.

Epidemiological studies and case reports of infectious diseases comprised relatively small proportions of the studies published in North Korean medical journals. This agrees with previous research suggesting that North Korean preventive medicine textbooks lacked information on the methods of epidemiological research [13], and that studies published in comprehensive medical research journals focused on the clinical characteristics, diagnosis, and treatment of diseases, while the scale of occurrence and risk factors were rarely discussed $[16,17]$. A reason for this may be North Korea's policy of avoiding any kind of disclosure of healthcare conditions, internally or externally. This study, nonetheless, was able to indirectly confirm through research trends that there are differences between South and North Korea in the prevalence in different diseases and in their occurrence patterns.

North Korea's research on TB was less focused on NTM, whereas more research was conducted on extrapulmonary TB and MDR-TB. This is most likely due to the fact that South Korea is following the international trend of increases in NTM compared to the decrease of TB [33]; in contrast, TB is still prevalent in North Korea, so North Korean researchers may be less interested in NTM. Some research has suggested a difference between South and North Korea in the frequencies with which different types of NTM are isolated. Although it is true that the credibility of research conducted in North Korea should be carefully considered, it is also necessary to investigate the causes for the epidemiological differences. Challenges exist in the management, storage, and sterilization of medi- 
cal equipment in North Korea, and traditional Korean medicine such as acupuncture, is very popular, so NTM diseases are expected to have a higher prevalence in North Korea, which makes epidemiological research even more important. Furthermore, a reason why North Korean medical journals publish a greater proportion of research on MDR-TB and extrapulmonary TB is that there are many patients with immunodeficiency who experience poor food conditions and malnutrition, and the relative amount of research into these diseases reflects their prevalence.

Research on parasitic diseases in North Korea showed less variety in the types of parasites, but some research was conducted on parasites that are no longer widespread in South Korea, especially hookworms and T. trichiura, providing an opportunity to analyze the conditions of parasitic diseases. This is in agreement with many previous studies that have estimated a high rate of T. trichiura infection in North Korea [8-10]. South Korea has been researching newly transmitted parasites from foreign countries, as it has successfully managed helminthic infections. North Korea, in contrast, is still focusing their research on the high-priority parasites. The fact that research on protozoa accounts for more than half of the studies conducted in North Korea seem to be related to the fact that malnutrition weakens the immune system, which increases the prevalence of protozoiasis. However, the statistics on protozoiasis in North Korea are unknown, so understanding the current conditions is an urgent matter.

In addition, it is estimated that the prevalence of clonorchiasis, T. gondii, and amoebas is high in North Korea. The main cause for clonorchiasis could be the dietary habit of eating raw fish, considering the conditions of North Korea's food sources and the difficulty of hygienic food storage. In fact, wild food is one of the most important food sources in North Korea; 89\% of households collect wild food and $30 \%$ of them consume wild food 2-3 times a week [34]. Furthermore, as an agrarian society, the North Korean population is predicted to have higher risks of being exposed to livestock infected with T. gondii; however, an accurate understanding of the situation is difficult because the number of reported patients is low and because it is asymptomatic. Amebiasis and cryptosporidiosis seem to be related to the poor conditions of sewage treatment facilities [4].

Hydatid and Cyclospora case reports in South Korea suggested that they were imported cases, while North Korean case reports have recorded indigenous cases. Therefore, a clear understanding of this situation is necessary to prepare for unification. There is a possibility that hydatids have been transmitted through livestock from China or Russia [35], and it is predicted that the endemic cases occurred from the storage of the distributed meats. Cyclospora has been determined to be a common cause of diarrhea in North Korea. However, the only case recorded in South Korea was an imported case, in a patient who had visited Indonesia in 2003 [36]. The fact that this parasite, which does not exist in South Korea, is being found to cause diarrhea in North Korea should also be considered in preparation for unification.

The articles analyzed in this study showed a great interest in North Korea in traditional Korean medicine as a way to overcome poor medical conditions. This confirms the findings of a previous study [17] that investigated North Korea's focus on practical research due to a lack of resources. Because laboratories that can conduct biochemical analysis and DNA tests for the diagnosis of TB are mostly centrally located, and may not be in suitable conditions for clinical use [37], several studies have sought to develop new methods to solve this problem. North Korea showed a particularly high percentage of research dedicated to treatment. Most of the studies were experimental studies based on traditional Korean medicine using natural products because, unlike South Korea, which has established TB treatment, North Korea must rely heavily on external sources for anti-TB drugs and has weaknesses in drug inventory management [37]. According to the testimonies of North Korean defectors, TB is not being properly treated because medical institutions do not follow prescription guidelines for anti-TB drugs or have patients purchase these drugs at private markets [38]. Additionally, an analysis of the medical journal Internal Medicine suggested that the basic medication for TB treatment in North Korea was streptomycin and isoniazid, with a treatment period ranging from 1 to 6 months [20]. The current conditions of TB treatment in North Korea suggest that drug-resistant TB has become more common in North Korea, as confirmed by the findings of this study that extensive research has been conducted into the treatment of drugresistant TB. Many studies focused on finding natural products that could replace metronidazole, which may also reflect the presence of insufficient amounts of antiprotozoals.

This study has certain limitations. First, the scope of the study only included the Jong Un Kim period, which means that its results are difficult to compare to previous time periods and attribute characteristics specific to the selected time 
period. Furthermore, infectious diseases other than TB, malaria, and parasitic diseases need to be analyzed, such as viral diseases, including hepatitis B. Second, it is difficult to claim that the trends observed in South Korea's medical journals completely represent South Korean researchers' interests. A systematic investigation of research trends in South and North Korea using an academic database in the future could supplement this limitation. Additionally, various methodologies have been used to identify recent research interests among North Korean researchers [16-20]. North Korean researchers have been submitting more studies to overseas journals, which may enable a more systematic comparison of research trends in South and North Korea.

Despite these limitations, this study was able to provide indirect evidence regarding the current conditions in North Korea, which is especially useful since direct investigations of infectious diseases in North Korea are difficult, and limited data exist due to the severance of exchange and cooperation between South and North Korea. Obtaining accurate information on the current conditions in North Korea is urgent because in addition to the relatively well-known diseases in North Korea, such as TB or malaria, less well-known diseases such as protozoiasis and NTM are estimated to have a high prevalence. Furthermore, unlike South Korea, which does not have indigenous cases of parasitic diseases such as Cyclospora and hydatids, North Korea is presumed to have such patients based on their studies, and this will become a critical problem when the two Koreas are unified.

In addition, the results of this study are useful for understanding the level and focus of medical research in North Korea. Medical knowledge and technology in North Korea do not seem to have fallen far behind, as they utilize molecular identification technologies in the diagnosis of pathogens. However, the research on the effects of natural products and PCRbased diagnoses indicates that it is difficult to apply these techniques in clinical practice immediately and universally. The fact that research focused primarily on diagnosis and treatment, rather than epidemiology, can be used to develop strategies for North-South Korea cooperation by reflecting the interests and priorities of North Korean researchers. Furthermore, the gap in the disease burden between South and North Korea can be resolved if treatment methods that can be applied to North Korean conditions are developed by understanding their strengths in traditional Korean medicine research and appropriately using the research results that have accumulated.

Infectious diseases will be a high-priority field when South and North Korea unify or in case of war or other disasters when large populations are in movement. The KCDC (2013) selected measles, TB, and waterborne microbial diseases as the prioritized infectious diseases to be regulated when there is a massive inflow of North Korean refugees, and pointed out that especially for TB, a prompt diagnosis and treatment protocol needs to be developed [39]. In preparation for this, the capacity to diagnose and treat diseases that have a high risk of spreading, such as TB and malaria, needs to be continually maintained [40] and countermeasures for public health crises need to be prepared if disasters do happen. Simultaneously, efforts must be made to strengthen North Korea's disease control system.

\section{CONFLICT OF INTEREST}

The authors have no conflicts of interest associated with the material presented in this paper.

\section{ORCID}

Do-Hyeon Park https://orcid.org/0000-0001-7294-5167

Min-Ho Choi https://orcid.org/0000-0002-0130-2815

Ah-Young Lim https://orcid.org/0000-0003-4445-5034

Hee Young Shin https://orcid.org/0000-0003-2091-1947

\section{REFERENCES}

1. World Health Organization. Global Health Observatory (GHO) data [cited 2017 Nov 27]. Available from: http://www.who.int/ gho/en/.

2. Ministry of Public Health. Medium term strategic plan for the development of the health sector in DPRK 2010-2015. Pyongyang: Ministry of Public Health; 2011, p. 52.

3. World Health Organization. Global tuberculosis report 2017 [cited 2017 Nov 27]. Available from: http://www.who.int/tb/ publications/global_report/en/.

4. United Nations Children's Fund. Situation analysis of children and women in the Democratic People's Republic of Korea 2017; 2016 [cited 2017 Nov 27]. Available from: https://www. unicef.org/dprk/Situation_Analysis_of_Children_and_Women_in_DPR_Korea_UNICEF_2017.pdf.

5. Shin YJ, Ki M, Sung N. A new strategy for tuberculosis control 
in North Korea. Epidemiol Health 2015;37:e2015053.

6. World Health Organization. World malaria report 2017; 2017 Nov 29 [cited 2018 Mar 13]. Available from: http://www.who. int/malaria/publications/world-malaria-report-2017/en/.

7. Park JW. Changing transmission pattern of plasmodium vivax malaria in the Republic of Korea: relationship with climate change. Environ Health Toxicol 2011;26:e2011001.

8. Hong ST, Yoon HW, Choi MH, Lee SO, Shin SH. Situation of parasitic infections in North Korea. Seoul: Seoul National University; 2008, p. 20 (Korean).

9. Li S, Shen C, Choi MH, Bae YM, Yoon H, Hong ST. Status of intestinal helminthic infections of borderline residents in North Korea. Korean J Parasitol 2006;44(3):265-268.

10. Kim SH. Strategies for the development of health system in North Korea through sustainable cooperation and development. Seoul: Ministry of Health and Welfare; 2008, p. 233 (Korean).

11. Lee SH, Kwon JE, Cheong YS. Two cases of Trichuris trichiura infection diagnosed by colonoscopy. Korean J Fam Med 2010; 31(8):622-629 (Korean).

12. Lim HJ, Lee G, Yang SJ. The trends in research on the health of North Korean refugees. J Korean Acad Community Health Nurs 2017;28(2):144-155 (Korean).

13. Yi SG, Moon OR, Lee SJ, Kim JC, Yong W, Lin PS. The state of the art of preventive medicine in North Korea with reference to the content analysis of a medical textbook. Korean J Prev Med 2000;33(3):373-382 (Korean).

14. Lee SG, Yoon HR, Lee GH, Moon OR. Medical technology of North Korea: with special reference to the content analysis of medical textbooks. Korean J Prev Med 1990;23(4):416-427 (Korean).

15. Kim OJ. An analysis of a North Korean medical journal: In-MinBo-Gun (People's Health) in 1950s. Korean J Med Hist 2002; 11(2):165-185 (Korean).

16. Huh S. Status of health in North Korea with reference to medical journals. Seoul: Ajou Institute of Korean Unification and Health Care; 2004, p. 181-227 (Korean).

17. Kim HJ, Noh SC. Theory and practice of preventive medicine in North Korean medical journals. Seoul: Ajou Institute of Korean Unification and Health Care; 2007, p. 1-30 (Korean).

18. Jung M, Chung D, Choi M. Keywords network analysis of articles in the North Korean Journal of Preventive Medicine 19972006. J Prev Med Public Health 2008;41(6):365-372 (Korean).

19. Kim SG. General characteristics and trends of North Korean medical journals. In: Proceedings of Fall Conference of the As- sociation of Healthcare for Korean Unification; 2016 Jul 1; Seoul. Seoul: Association of Healthcare for Korean Unification; 2016, p. 79-98 (Korean).

20. Choi SI, Lee EJ, Jeong WJ, Min KH, Heo KY, Lee SH, et al. P-57 recent 10 years of medical research trends in North Korea, focused on tuberculosis and respiratory diseases research published in North Korean medical journals. In: Proceedings of Fall Conference of the Korean Academy of Tuberculosis and Respiratory Diseases; 2016 Nov 17; Seoul. Seoul: Korean Academy of Tuberculosis and Respiratory Diseases; 2016, p. 201 (Korean).

21. Kwangmyong encyclopedia of North Korea 7 education, language and media. Pyongyang: Encyclopedia Publisher; 2011, p. 729-735 (Korean).

22. Encyclopedia of North Korea. Pyongyang: Encyclopedia Publisher; 2001 (Korean).

23. Jeong GH, Huh S. Bibliometric and content analysis of medical articles in the PubMed database published by North Korean authors from 1997 to July 2017. Sci Ed 2017;4(2):70-75.

24. Pant SD, Chol KY, Tegegn Y, Mandal PP, Chol RK. Mass primaquine preventive treatment for control of Plasmodium vivax malaria in the Democratic People's Republic of Korea: a country success story. WHO South East Asia J Public Health 2014; 3(1):75-80.

25. Koh WJ, Kwon OJ, Jeon K, Kim TS, Lee KS, Park YK, et al. Clinical significance of nontuberculous mycobacteria isolated from respiratory specimens in Korea. Chest 2006;129(2):341-348.

26. Ryeo MC, Kim SC, Kim JC. Up-to-date diagnosis of pulmonary TB and evaluation of disease activity. Preventive Medicine 2012;(1):52-53 (Korean).

27. Kim SY. The use of phage display in malaria research. Basic Medicine 2013;(1):56 (Korean).

28. Lee EB. Lee EH. Epidemiological study of biliary ascariasis. Preventive Medicine 2016;(3):25-26 (Korean).

29. Byun SJ, Moon KC, Suh KS, Han JK, Chai JY. An imported case of echinococcosis of the liver in a Korean who traveled to western and central Europe. Korean J Parasitol 2010;48(2): $161-165$

30. Yoon HJ, Ji CS. Assessment of Cryptosporidium's ability to survive using mouse infectivity test. Preventive Medicine 2016; (2):27 (Korean).

31. Kim YS, Kim SS. Epidemiological characteristics of Cyclospora from some regions. Preventive Medicine 2015;(2):14 (Korean).

32. Kim W, Jo HK. Detection of Toxoplasma gondii in meat samples using a real-time PCR. Basic Medicine 2015;(3):56 (Korean). 
33. Park YS, Lee CH, Lee SM, Yang SC, Yoo CG, Kim YW, et al. Rapid increase of non-tuberculous mycobacterial lung diseases at a tertiary referral hospital in South Korea. Int J Tuberc Lung Dis 2010;14(8):1069-1071.

34. World Food Programme. Performance assessment of "nutrition support to women and children" PRRO 200114: delivering nutrition support in the Democratic People's Republic of Korea; 2013 [cited 2017 Dec 15]. Available from: https://www. wfp.org/sites/default/files/PRRO\%20200114\%20end-of-project\%20review_report_FINAL_27.11.2013.pdf.

35. Grosso G, Gruttadauria S, Biondi A, Marventano S, Mistretta A. Worldwide epidemiology of liver hydatidosis including the Mediterranean area. World J Gastroenterol 2012;18(13):14251437.

36. Yu JR, Sohn WM. A case of human cyclosporiasis causing traveler's diarrhea after visiting Indonesia. J Korean Med Sci 2003;
18(5):738-741.

37. Ministry of Public Health. National strategic plan for tuberculosis control 2015-2018. Pyongyang: Ministry of Public Health; 2014, p. 20-25.

38. Korea Foundation for International Healthcare. Building midterm strategies for tuberculosis management support towards North Korea. Seoul: Korea Foundation for International Healthcare; 2015, p. 83 (Korean).

39. Korea Centers for Disease Control and Prevention. Strategy development of public health emergency response system for natural and manmade disaster. Cheongju: Korea Centers for Disease Control and Prevention; 2013, p. 293 (Korean).

40. Nishiura $H$, Lee $H$, Yuan B, Endo A, Akhmetzhanov AR, Chowell $\mathrm{G}$. Infectious disease risks among refugees from North Korea. Int J Infect Dis 2018;66:22-25. 